\title{
Treatment of atlantoaxial instability in pediatric patients
}

\author{
Scott Y. Rahimi, M.D., E. Andrew Stevens, B.S., David John Yeh, M.D., \\ ann Marie Flannery, M.D., Haroon Fiaz Choudhri, M.D., \\ ANd Mark R. Lee, M.D., Ph.D.
}

Department of Neurosurgery, Medical College of Georgia, Augusta, Georgia

\begin{abstract}
The atlantoaxial region has been extensively described as a spinal segment especially prone to injury in children. In this clinical review, the authors evaluate and summarize the management of 23 pediatric cases of atlantoaxial instability treated between March 1990 and October 2002. Four broad categories of atlantoaxial problems were observedatlantoaxial rotatory subluxation in six patients, anterior-posterior atlantoaxial instability caused by ligamentous injury or congenital ligamentous laxity (10 patients), atlantoaxial fracture with or without dislocation (five patients), and atlantooccipital dislocation (two patients). Most cases (60.9\%) were treated without surgical intervention, resulting in excellent outcomes; however, $21.7 \%$ of cases were treated with a cervical halo (mean patient age 72.6 months) alone for 3 months. Various techniques of surgical stabilization including transarticular screws with sublaminar wiring, transoral decompression with posterior plating, and laminectomy with Steinmann pin occipital-cervical fusion were used with good results. Both patients with atlantooccipital dislocation underwent immediate Locksley occipital-cervical fusion, with marked neurological improvement. Individualized case management must be based on clinical presentation, with internal fixation being the last resort.
\end{abstract}

\section{KEY WORDS • atlantoaxial injury • atlantoaxial stabilization $・$ cervical spine}

The spinal cord is most frequently injured at the cervical level in all pediatric age groups. ${ }^{3,4,10}$ Most spinal injuries in children younger than 8 years of age occur above the level of C-4, whereas in older children fractures/dislocations more commonly involve the lower cervical spine. ${ }^{3}$ The atlantoaxial region in pediatric patients has several well-described characteristics that predispose it to injury: 1) increased ligamentous laxity; 2) more horizontally oriented facets; 3) less mature bone ossification; 4) higher fulcrum of cervical movement; and 5) higher inertia and torque forces associated with a larger head/body mass ratio. $^{2-5,9,10}$ The optimal management of atlantoaxial problems in children remains controversial. ${ }^{7}$

\section{CLINICAL MATERIAL AND METHODS}

We reviewed 2545 available clinical charts obtained between March 1990 and October 2002 and identified 23 cases of atlantoaxial instability in children (12 boys and 11 girls) between the ages of 1.8 and 14.6 years (mean age 7.5 years). Only five other severe cervical injuries had occurred: two cases of C2-3 subluxation, and one case each of C7-T1 fracture, C-6 fracture, and C6-7 fracture associated with Klippel-Feil syndrome. The most common cause of atlantoaxial instability was trauma $(72.7 \%)$. Congenital anomalies resulting from Down syndrome, spon-

Abbreviations used in this paper: $\mathrm{CT}=$ computerized tomography; $\mathrm{MR}=$ magnetic resonance. dyloepiphysial and spondylometaphysial dysplasia, and other unspecified genetic conditions leading to atlantoaxial instability occurred in six cases $(26.1 \%)$. One case of atlantoaxial rotatory subluxation resulting from Grisel syndrome was seen. The mean patient follow up was 10 months (range 2 weeks-54 months), with only two patients being lost to follow up after an initial 2-week posthospitalization clinic visit.

\section{RESULTS}

Four broad categories of atlantoaxial problems were identified.

\section{Atlantoaxial Rotatory Subluxation}

Six patients had atlantoaxial rotatory subluxation. Four patients had presented with torticollis and two with severe neck pain. The causes of these disorders were trauma $(83 \%)$ and infection (17\%). Dynamic CT scanning is ideally suited for the evaluation of atlantoaxial rotatory subluxation. In younger patients, however, accurate dynamic studies were not always possible because of pain noncompliance, thus further complicating this difficult diagnosis. ${ }^{6}{ }^{12}$ Most cases of atlantoaxial rotatory subluxation reduced spontaneously with the use of a rigid cervical collar $(50 \%)$ or with 48 to 72 hours of traction followed by the use of a collar (33\%). The time between symptom onset and the application of a cervical brace was less than 24 hours in four cases and 11 days and 53 days in the other 

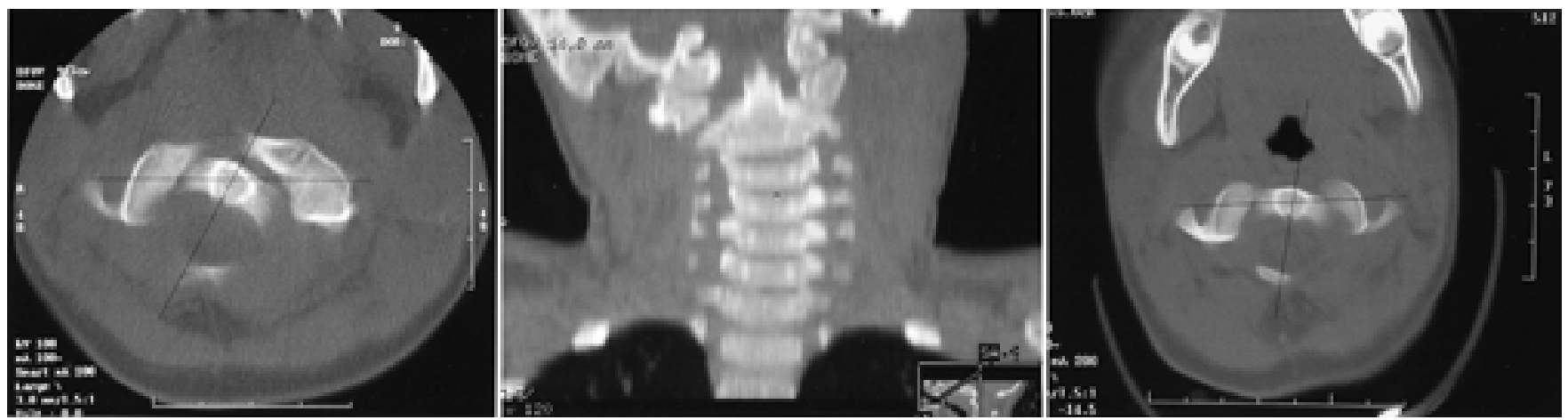

Fig. 1. Computerized tomography scans obtained in a 2.5-year-old girl with torticollis following severe respiratory tract infection with lymphadenopathy. Her condition improved after 8 days in a cervical collar; however, she developed severe recurrent symptoms 3 weeks later while playing on a slide. She was unable to tolerate dynamic CT scanning. Left and Center: Axial and coronal reconstructed CT scans of the neck illustrating a cock-robin deformity, with the head rotated to the left and laterally flexed to the right. Right: Repeated CT scan of the neck obtained after treatment with 72 hours of cervical sling traction and 4 weeks of fitted cervical bracing, showing restoration of normal atlantoaxial alignment in the neutral position. The collar was removed after 3 months, with complete resolution of the torticollis.

two cases. One severe case of traumatic atlantoaxial rotatory subluxation with a small spinal canal epidural hematoma and a right hemiparesis was treated with the use of a cervical halo for 3 months, resulting in a full neurological recovery. One patient was treated with traction and bracing following a recurrence (Fig. 1).

\section{Atlantoaxial Ligamentous Instability}

Ten patients had atlantoaxial ligamentous instability. Six had presented with congenital abnormalities and four with traumatic injuries. Half of the patients required surgical stabilization, and two who presented with progressive myelopathy required decompression prior to arthrodesis (Fig. 2). Cervical spine $\mathrm{x}$-ray films, with flexion and extension views demonstrating movement at the preodontoid space, were most helpful in diagnosing anteroposterior atlantoaxial instability. Additionally, MR imaging studies performed in traumatic cases was helpful in identifying ligamentous injury. Two patients (20\%) were treated conservatively with observation, and three patients (30\%) required external orthoses only, that is, two with halo fixation (3 months) and one with a cervical collar alone (6 weeks).

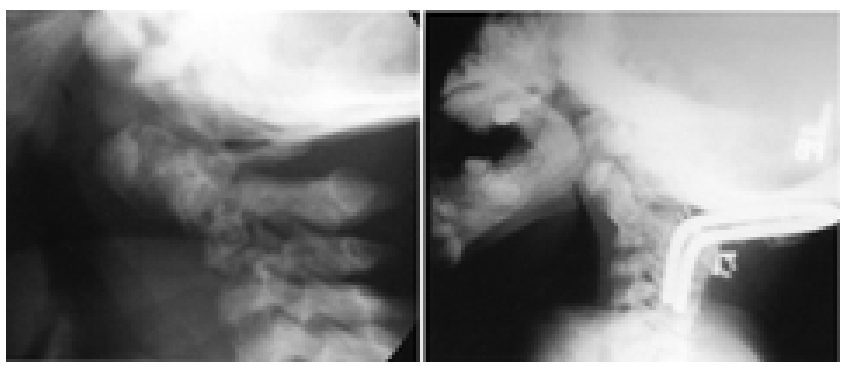

Fig. 2. Preoperative (left) and postoperative (right) x-ray films obtained in a 4-year-old girl with multiple congenital abnormalities and progressive myelopathy from craniocervicomedullary compression after foramen magnum and C-1 decompression with occiput-C2 fusion.

\section{Traumatic Atlantoaxial Fracture}

Five patients had traumatic atlantoaxial fracture. Two patients were treated with halo fixation for 3 months: one C1-2 displaced fracture (reduced under anesthesia) and one acute Type II odontoid fracture. A C-2 nondisplaced burst fracture was treated using a cervical collar $(8$ weeks), with good results. One case of a C-1 fracture was treated conservatively with the aid of a collar. One C1-2 fracture/dislocation was treated with transarticular screws and sublaminar wiring (Fig. 3).

\section{Atlantooccipital Dislocation}

Two cases of traumatic atlantooccipital dislocation occurred in patients with a mean age of 11.5 years. Both patients presented with closed head injury and cranial nerve palsies. Diagnoses were made with the aid of plain x-ray films. ${ }^{8,9,11,13}$ Immediate occiput-C4 Locksley fusion with an autograft rib was performed in both cases (Fig. 4). No external orthoses were applied postoperatively. Both patients improved remarkably, with resolution of cranial nerve deficits and functional recovery.

\section{Treatment Complications}

Two treatment complications $(9 \%)$ occurred. One patient with Down syndrome who had undergone C1-2 transarticular screw fusion for chronic os odontoideum developed a chronic wound infection and required revision 4 months postoperatively. One case of osteomyelitis at the halo pin site in a 22-month-old required surgical debridement.

\section{DISCUSSION}

Pediatric spinal cord and spinal column injuries are relatively uncommon: the frequency of these types of injuries in the current literature ranges from 1 to $10 \% .^{3} \mathrm{Sev}-$ eral factors differentiate the incidence, type, and location of spine injuries that occur in children compared with those occurring in adults. This variance is largely attrib- 


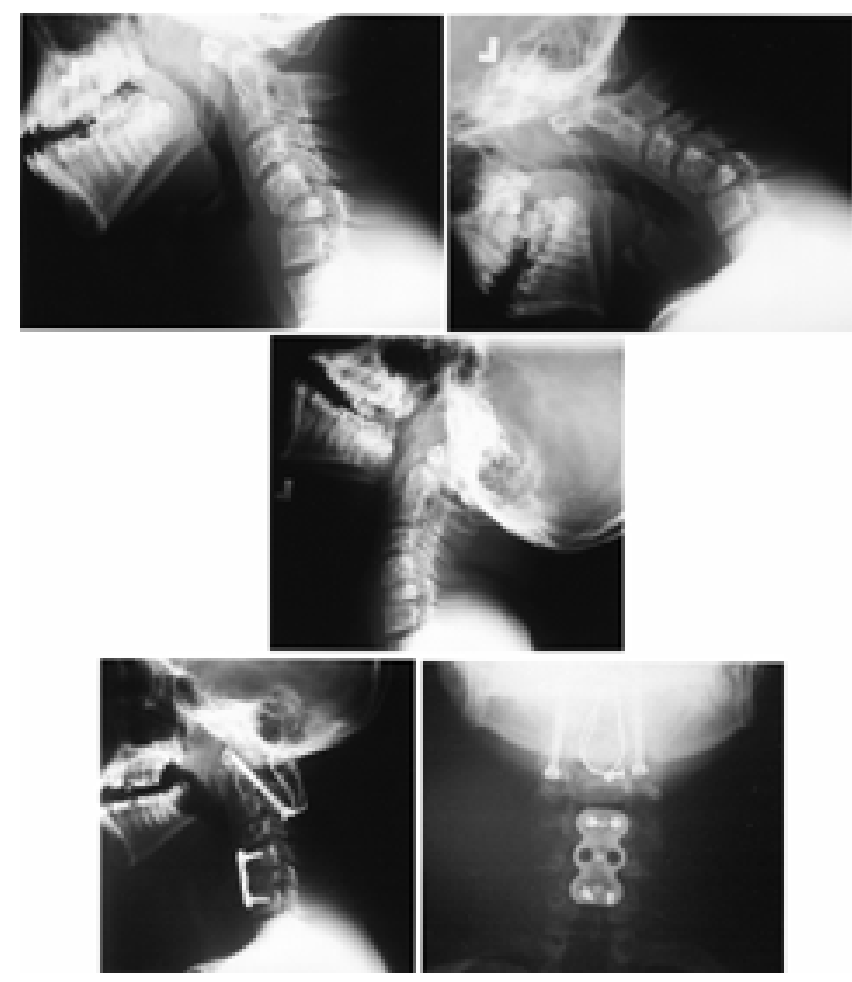

Fig. 3. Several x-ray films obtained in a 14-year-old boy following a traumatic motor vehicle accident, demonstrating a Type II odontoid fracture as well as a C-5 fracture and an unstable kyphotic deformity. After 2 months in a cervical collar, he presented with persistent neck pain. Upper Left: Neutral x-ray film showing a C-5 vertebral body compression fracture with an increased atlantodental interval. Upper Right and Center: Flexion extension films showing marked atlantoaxial and $\mathrm{C} 4-5$ instability. Lower Left and Lower Right: Postoperative films obtained after $\mathrm{C} 1-2$ transarticular screw placement with posterior laminar wiring and C-5 anterior cervical corpectomy with plating. Three months later the patient had no complaints, x-ray films demonstrated stable fusion, and the collar was removed.

uted to anatomical and biomechanical factors in the developing pediatric spine. Such factors include the disproportionately large head mass and underdeveloped neck musculature in the pediatric population, which contribute to greater bending of the cervical spine with flexion/extension forces; greater elasticity of the interspinous and posterior joint capsules, which allow for hypermobility; horizontally angulated articulating facets, especially at the C1-2 level; and wedge-shaped vertebral bodies that allow for anterior sliding. ${ }^{2}$ Due to the aforementioned factors, pediatric spine injuries most often involve the cervical spine (specifically, above the level of C-3) ${ }^{1}$ Not until approximately the age of 8 years will a child's cervical spine begin to form the characteristics of an adult spine. At that point, the ligaments and facet capsules gain more tensile strength, facet joints become more vertically aligned, and cervical musculature becomes more developed.

In our series, we identified 28 children with cervical spine injuries. Twenty-three $(82 \%)$ of these injuries involved atlantoaxial instability and five $(18 \%)$ pancervical injuries below the C-2 level. The majority of children with atlantoaxial instability in our series $(65 \%)$ were treated conservatively. Such methods included no intervention (two cases), halos (five cases), and cervical collars (eight cases). Neurosurgical procedures were needed in eight cases.

The initial evaluation of patients with neck pain and suspected atlantoaxial injury begins with obtaining plain x-ray films (Fig. 5). Children with neurological deficits should immediately be placed in cervical collars for immobilization. Plain x-ray films demonstrating nondiagnostic findings or those positive for atlantoaxial injury should be followed by CT scanning. Dynamic CT scanning is very useful in these instances. Note, however, that many pediatric patients may be unable to tolerate these procedures. A CT scan that reveals an atlantoaxial injury would justify performing further imaging with MR studies. An injury that appears to be stable on MR images may be treated conservatively with collar immobilization or traction (sling). An MR image revealing an unstable injury should cue one to treat the patient by surgical intervention immediately. Several treatment options are available in patients who demonstrate sustained atlantoaxial injuries on imaging studies (Fig. 6). Patients with atlantooccipital dislocation require urgent fusion procedures. Different $\mathrm{C}-1$ or C-2 fractures may be treated in several ways. An unstable injury requires immediate surgical intervention. With stable C-1 or C-2 injuries, however, conservative treatment with a cervical halo or a cervical col-

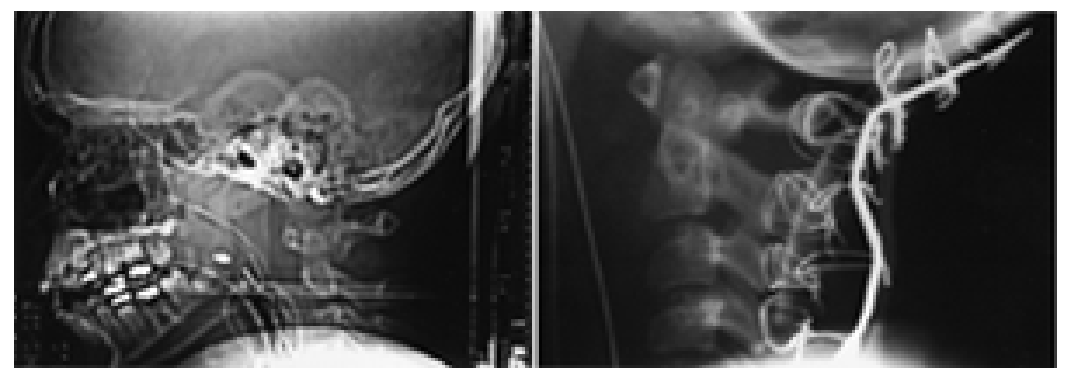

Fig. 4. Left: Lateral cervical spine x-ray film obtained in a 10-year-old girl who had been in a motor vehicle accident and presented with left abducens palsy and quadriparesis, showing separation of occipital condyles from the lateral masses of C-1, widening of the C1-2 interspace, and paravertebral soft-tissue swelling around C-2. Right: An x-ray film demonstrating Locksley fusion with autograft rib, titanium plate, and sublaminar wires. The 2-year follow-up examination revealed full strength in all extremities and extraocular muscles, with only a slight limp. 


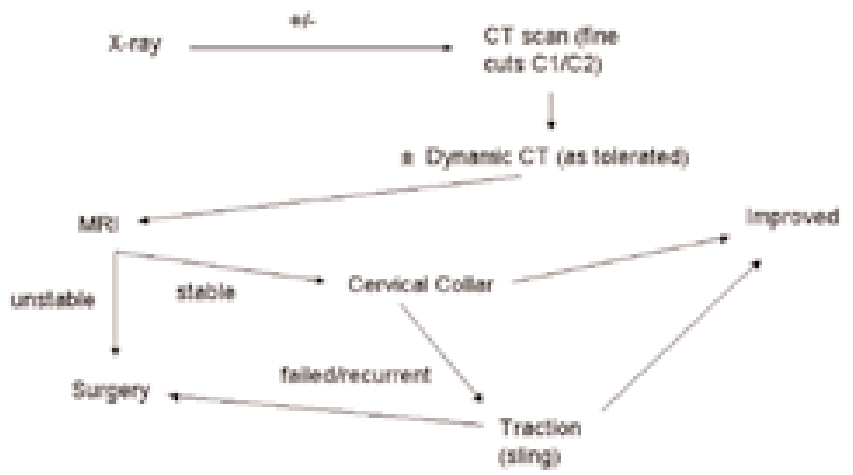

Fig. 5. Schematic illustrating the evaluation process in patients with neck pain and suspected atlantoaxial injury, but no neurological deficits.

lar may be appropriate. Atlantoaxial rotatory subluxation injuries may initially be treated using cervical traction (sling) for 72 hours. On repeated imaging studies, injuries that demonstrate no reduction will require surgical intervention. Injuries that appear to be reduced can be treated using a collar together with follow-up imaging.

All patients who underwent surgical and conservative treatment in our series achieved good recoveries. A total of two complications were noted during our review, as mentioned earlier. The complication rate in the groups treated conservatively and surgically were the same. Different diagnostic modalities were used in assessing the patients with atlantoaxial instability. Dynamic CT scanning was ideally suited for the evaluation of atlantoaxial rotatory subluxation. ${ }^{11}$ Computerized tomography scanning was also ideal for the detection of atlantoaxial fractures. Ligamentous injuries were best assessed on MR imaging studies in all instances. The initial diagnosis of atlantooccipital dislocation in two patients was made with the aid of plain x-ray films, which are usually sufficient to make the diagnosis. ${ }^{7}$ Magnetic resonance imaging was crucial in further assessment and treatment of these injuries.

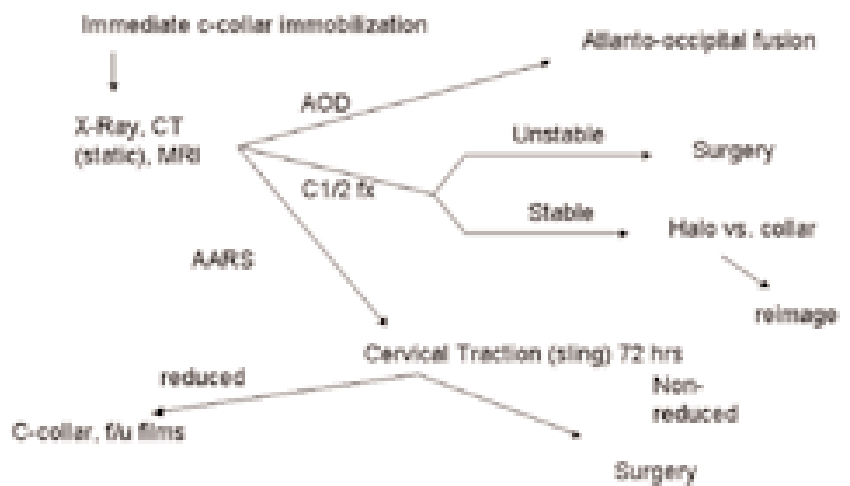

Fig. 6. Schematic illustrating the evaluation process in patients with neck pain and suspected atlantoaxial injury, with neurological deficits. AARS = atlantoaxial rotatory subluxation; $\mathrm{AOD}=$ atlantooccipital dislocation; $\mathrm{c}=$ collar $=$ cervical collar; $\mathrm{f} / \mathrm{u}=$ follow-up; fx = fracture.

\section{CONCLUSIONS}

Atlantooccipital injuries are commonly seen among pediatric spine injuries. When surgical intervention is indicated, we have used different fusion methods-all with excellent outcomes. The following conclusions are indicated by our data in this series. 1) Halo fixation is a welltolerated option for conservative management, but is not complication-free, especially in very young patients. ${ }^{7} 2$ ) Dynamic imaging is critical to properly assessing patients with continued complaints of neck pain. 3) Patients with atlantoaxial rotatory subluxation most often present with painful torticollis. Diagnosis is controversial; however, most cases resolve with the use of cervical bracing for time periods shorter than 6 weeks. 4) A high index of suspicion may improve outcome in patients surviving atlantooccipital dislocation. Immediate occipital-cervical fusion can result in significant neurological improvement. 5) Children with developmental abnormalities-KlippelFeil syndrome, Down syndrome, spondyloepiphysial/ spondylometaphysial dysplasia, and so forth-have an increased risk of atlantoaxial instability.

\section{References}

1. Fesmire FM, Luten RC: The pediatric cervical spine: developmental anatomy and clinical aspects. J Emerg Med 7: 133-142, 1989

2. Givens TG, Polley KA, Smith GF, et al: Pediatric cervical spine injury: a three-year experience. J Trauma 41:310-314, 1996

3. Hadley MN, Zabramski JM, Browner CM, et al: Pediatric spinal trauma. Review of 122 cases of spinal cord and vertebral column injuries. J Neurosurg 68:18-24, 1988

4. Heffez DS, Ducker TB: Fractures and dislocations of the pediatric spine, in Pang D (ed): Disorders of the Pediatric Spine. New York: Raven Press, 1995, pp 517-529

5. Hill SA, Miller CA, Kosnik EJ, et al: Pediatric neck injuries. A clinical study. J Neurosurg 60:700-706, 1984

6. Li V, Pang D: Atlanto-axial rotatory fixation, in Pang D (ed): Disorders of the Pediatric Spine. New York: Raven Press, 1995, pp 531-553

7. Pang D: Principles and pitfalls of spinal stabilization in children, in Pang D (ed): Disorders of the Pediatric Spine. New York: Raven Press, 1995, pp 575-604

8. Papadopoulos SM, Dickman CA, Sonntag VKH, et al: Traumatic atlantooccipital dislocation with survival. Neurosurgery 28:574-579, 1991

9. Powers B, Miller MD, Kramer RS, et al: Traumatic anterior atlanto-occipital dislocation. Neurosurgery 4:12-17, 1979

10. Ruge JR, Sinson GP, McLone DG, et al: Pediatric spinal injury: the very young. J Neurosurg 68:25-30, 1988

11. Sponseller PD, Cass JR: Atlanto-occipital fusion for dislocation in children with neurologic preservation. A case report. Spine 22:344-347, 1997

12. Subach BR, McLaughlin MR, Albright AL, et al: Current management of pediatric atlantoaxial rotatory subluxation. Spine 23:2174-2179, 1998

13. Traynelis VC, Marano GD, Dunker RO, et al: Traumatic atlanto-occipital dislocation. Case report. J Neurosurg 65:863-870, 1986

Manuscript received August 18, 2003.

Accepted in final form November 12, 2003.

Address reprint requests to: Scott Y. Rahimi, M.D., Department of Neurosurgery, Medical College of Georgia, 1120 15th Street, BI3088, Augusta, Georgia 30912. email: SRAHIMI@ mail.mcg.edu. 